\title{
Une centralisation renforcée par le chiffre? Les effets contradictoires des indicateurs chiffrés dans la police nationale en France
}

\author{
Anne-Cécile Douillet ${ }^{a}$, Jacques de Maillard ${ }^{b *}$ \\ et Mathieu Zagrodzkic \\ aUniversité de Lille-CERAPS \\ ' UVSQ-CESDIP et Institut Universitaire de France \\ 'UVSQ-CESDIP
}

\section{Résumé}

Les indicateurs chiffrés ont pris une importance croissante au sein de la Police Nationale dans un contexte d'introduction du nouveau management public et de recours croissant aux nouvelles technologies. Au-delà de l'usage politique de ces chiffres, l'article s'interroge sur leurs effets concrets sur les pratiques et des priorités de la police, en particulier sur un éventuel renforcement du caractère centralisé de l'organisation policière. Le poids grandissant des chiffres s'est traduit par une utilisation accrue de différentes bases de données comme la Main Courante Informatisée et autres tableaux de bord, par l'introduction de primes à la performance ainsi que par un renforcement des équipes chargées de collecter et de transmettre les données. La nécessité de rendre des comptes et de faire remonter l'information à tous les échelons de la hiérarchie, du commissariat au ministère, a contribué à un renforcement de la pyramide hiérarchique tout en réduisant la marge d'autonomie des agents. Ceux-ci ressentent une forme de frustration face à des demandes de plus en plus pressantes d'information et de résultats émanant de la hiérarchie. En découlent un certain nombre de stratégies d'évitement, d'adaptation, voire de tricherie, afin de fournir de bons chiffres en réponse aux objectifs assignés par le niveau supérieur, souvent suspecté de se soucier uniquement des statistiques au détriment de l'efficacité du « vrai » travail policier. Les effets centralisateurs sont ainsi contrebalancés par des pratiques centrifuges.

(c) 2014 IDMP/Lavoisier SAS. Tous droits réservés

Mots clés : police, management, centralisation, indicateurs, chiffres, statistiques.

\footnotetext{
*Auteur correspondant : demaillard@cesdip.fr

doi :10.3166/pmp.31.421-442 @ 2014 IDMP/Lavoisier SAS. Tous droits réservés
} 


\section{Abstract}

\section{A centralisation reinforced by numbers? Uses and effects of quantified indicators} in the French Police nationale. Quantified indicators have become increasingly important within the Police nationale as a result of the introduction of new public management and the growing uses of information technologies. It is necessary to examine the specific effects of these figures on the practices and priorities of the police, especially on a possible strengthening of the centralized nature of the police organization. The growing weight of numbers has resulted in increased use of various databases such as the Main courante informatisée and other dashboards, the introduction of performance bonuses as well as a strengthening of teams in charge of data collection, transmission and analysis. The need for accountability at all levels of the hierarchy has contributed to a reinforcement of the hierarchical nature of the police while reducing the margin of autonomy of agents. The latter feel a form of frustration towards centralised requests for urgent information and results. As a consequence, one may observe a variety of avoidance, adaptation or gaming strategies aiming at providing good numbers and achieving the objectives of the upper level which is often suspected of worrying about statistics at the expense of the efficiency and effectiveness of "real" police work.

Keywords: police, management, centralisation, indicators, numbers, statistics.

\section{Introduction}

Comme dans bien d'autres administrations publiques ${ }^{1}$, les indicateurs chiffrés ont pris une importance grandissante ${ }^{2}$ au sein de la police au cours des dernières décennies. Ces chiffres visent à la fois à mieux connaître l'état et l'usage des moyens et à mieux apprécier l'activité et les résultats des services. Leur mobilisation accrue est en grande partie la traduction du souci affiché de rendre la police plus performante en lui assignant des objectifs et en contrôlant ses résultats. Cet usage renforcé des indicateurs chiffrés pour mesurer et piloter l'activité policière est en effet directement lié à l'essor du nouveau management public qui, en France comme ailleurs, a pénétré le monde policier (Jones et Newburn, 2009; Maillard, 2009), et dont l'un des préceptes est la mise en place d'une « gestion par les résultats fondée sur la réalisation d'objectifs, la mesure et l'évaluation des performances et de nouvelles formes de contrôle » (Bezes, 2012 : 17). Les indicateurs chiffrés apparaissent particulièrement adaptés à la gestion par les résultats et à la comparaison des « performances » dans la mesure où le chiffre, comme les normes ou les procédures, constitue « une abstraction bureaucratique » (Hibou, 2012 : 43), une convention pour simplifier et appréhender le réel; la mise en chiffres correspond à une opération d'agrégation, reposant sur la construction de nomenclatures englobantes, en même temps qu'à une forme de réduction de la complexité, réduisant des situations humaines plurielles dans des catégories statistiques. «Le chiffre » permet ainsi aux acteurs de se mesurer, d'échanger, de se comparer.

\footnotetext{
${ }^{1}$ Sur l'hôpital, voir par exemple (Belorgey, 2010); sur l'enseignement supérieur et la recherche: (Bruno, 2008).

${ }^{2}$ La mesure de l'activité policière par des indicateurs chiffrés n'est en effet pas complètement nouvelle (cf. infra).
} 
Ces chiffres alimentent de nombreux débats et controverses. L'usage politique des statistiques de la délinquance est ainsi régulièrement dénoncé, aussi bien par des observateurs académiques $^{3}$ que par les acteurs politiques eux-mêmes ${ }^{4}$. Ces critiques de l'instrumentalisation des chiffres peuvent d'ailleurs s'appuyer sur des analyses approfondies de la fabrique des statistiques de police et de gendarmerie (Matelly et Mouhanna, 2007), qui soulignent le caractère «partiel, biaisé et insuffisant» (Le Bouillonnec et Quentin, 2013 : 11) des données. Quoi qu'il en soit, malgré leur faible crédibilité et les critiques récurrentes dont elles sont l'objet, la mobilisation de ces statistiques par les acteurs médiatiques et politiques de tout bord perdure, du fait en particulier d'un « sens politique commun en matière de sécurité » (Cos, 2012).

La mobilisation politique des « chiffres de la police »n'épuise pas cependant la question de l'usage de ces indicateurs. Se pose aussi la question de leur maniement par l'organisation et les acteurs policiers et d'un possible gouvernement des activités policières par les indicateurs chiffrés : comment les différentes composantes de l'institution policière utilisent-elles les indicateurs chiffrés et quelles significations leur donnent-elles; quels sont les effets sur les pratiques et les relations de travail? Il convient alors de regarder de près comment l'action policière est appréhendée sur la base de tableaux de bords, agrégats statistiques et comptes rendus d'activités, comment ces données sont lues et comment cela affecte l'action policière. Dans cette perspective, un certain nombre de travaux ont avancé que les obligations de bons résultats auraient conduit en France à une «politique du chiffre », c'est-à-dire à des pratiques policières privilégiant le court terme, les affaires faciles et le trucage statistique (Mucchielli, 2008 ; Matelly et Mouhanna, 2007), pour produire des statistiques conformes aux attentes gouvernementales. Le respect des objectifs quantitatifs se ferait ainsi aux dépens de la qualité du travail policier, ce que soutiennent aussi certaines positions politiques ou syndicales. En Angleterre également, l'utilisation des indicateurs de performance a suscité une abondante littérature sur les effets pervers de la pression statistique, qu'il s'agisse du resserrement du mandat policier autour de ce qui se mesure, des multiples formes de jeux avec les chiffres, ou des effets négatifs sur le moral des agents (Fitzgerald et al., 2002 ; Hough, 2007; Loveday, 2006). Quant au «miracle new yorkais », il a été mis en question par des travaux qui ont souligné à la fois la pression hiérarchique associée à l'obligation de résultats chiffrés et les pratiques de manipulation qui en ont résulté (Eterno et Silverman, 2012).

Dans le cadre de cet article, c'est à travers la question des effets sur l'organisation policière que nous nous proposons d'analyser ce que les indicateurs chiffrés font au travail policier. Ce qui est interrogé ici, c'est moins le travail des policiers sur le terrain, et l'effet

\footnotetext{
${ }^{3}$ Voir par exemple: Laurent Mucchielli, «Délinquance: la com' rituelle du ministère de I'Intérieur », 21 janvier 2011, Médiapart.

${ }^{4}$ Suite à une déclaration de Brice Hortefeux sur le nombre de voiture brûlées à la St Sylvestre, Manuel Valls, ministre de l'intérieur, déclare ainsi : «Contrairement aux propos de M. Hortefeux, la délinquance ne se combat pas à coups de statistiques élaborées selon des méthodes incomplètes ou fluctuantes. II faut sortir des postures d'instrumentalisation. » (Libération, « Délinquance : la valse des chiffres », 3 janvier 2013)
} 
des indicateurs de performance sur leur «pouvoir discrétionnaire $»^{5}$, que les effets sur les relations hiérarchiques : les indicateurs chiffrés relèvent-ils plus d'une forme de contrôle et de pilotage par le centre ou favorisent-ils l'autonomisation des services aux différents niveaux de la hiérarchie ? Ces questionnements ont une dimension organisationnelle évidente et, dans le cas français, la question des effets (dé)centralisateurs des objectifs chiffrés se pose d'autant plus que l'organisation policière est marquée par une longue tradition de centralisation : les réformes managériales transforment-elles la structure de l'administration de la police vers une plus grande déconcentration; comment affectent-elles les relations entre les différents corps (conception et direction/commandement/encadrement et application) qui composent la police? Mais ces questionnements renvoient aussi à des enjeux plus politiques : il s'agit de mieux saisir à la fois les modalités de définition des objectifs policiers (où se définissent les orientations et les priorités ?) et la façon dont les policiers rendent compte de leur activité (que donnent-ils à voir de leur action à travers les indicateurs chiffrés ?). Une des questions sous-jacentes est ainsi celle de la transparence : ces indicateurs permettentils une moindre opacité de l'organisation policière, non seulement vis-à-vis d'elle-même (permettent-ils aux agents de mieux appréhender le travail des différents services ?) mais aussi vis-à-vis de l'extérieur?

La littérature managériale associe « gestion par les résultats » et responsabilisation de tous les échelons (Biondi et al., 2008), associant implicitement ce mode de management à un mode «participatif », ou du moins déconcentré, de définition des objectifs. Gestion par les résultats et gestion déconcentrée seraient ainsi quasi synonymes, l'efficacité d'une gestion plus décentralisée (les objectifs seraient d'autant plus facilement atteints qu'ils sont définis avec ceux qui doivent les atteindre) justifiant en quelque sorte l'introduction d'une logique de gestion par les résultats. Pourtant, la plupart des études sur les réformes managériales dans la police concluent à une centralisation accrue, en raison de l'imposition des mêmes objectifs et priorités à des unités pourtant différentes mais aussi du fait des contrôles accrus et des comptes que les échelons subalternes doivent rendre à leurs supérieurs (Collier, 2006; Hough, 2007). Ces travaux soulignent par ailleurs que la logique de reddition de compte (accountability) tend à changer de nature : elle devient moins « communautaire » (en direction de la population) et plus «managériale » (en direction de l'encadrement) (Loveday, 2006). Le recours aux indicateurs chiffrés apparaît ainsi comme relevant d'une logique descendante (top down) : les objectifs sont définis au niveau national, de même que l'allocation des ressources, ce qui s'accompagne de la généralisation des contrôles et alimente un cercle vicieux d'intervention de plus en en plus directe des niveaux supérieurs. Analysant de façon plus générale la diffusion de la logique de « gestion par résultats », B. Hibou (2012, p. 113) conclut pour sa part de façon radicale que « le principe d'efficacité, la logique de performance et la gestion par résultat - qui nécessitent ${ }^{6}$ la centralisation des

\footnotetext{
${ }^{5}$ Sur ce point, la mesure de la performance des policiers et la fixation d'objectifs ont été analysés comme un mode d'encadrement ou de réduction du pouvoir discrétionnaire du fonctionnaire de terrain (Savage et Golding, 2008). Dans les entretiens que nous avons réalisés, certains commissaires reconnaissent avoir parfois incité à « faire des ILS » (infractions à la législation sur les stupéfiants) ou des ILE (infractions à la législation sur les étrangers) tandis que les gradés et gardiens de la paix évoquent ici et là une « pression à l'interpellation ». Ces pressions et incitations apparaissent cependant très variables dans le temps et dans l'espace; il est par ailleurs difficile d'en apprécier la portée et les effets sur la base des seuls entretiens.

${ }^{6}$ C'est nous qui soulignons.
} 
décisions et un exercice autoritaire du pouvoir - trouvent en effet leur puissance dans cette omniprésence de la quantification ».

Pour le cas de la police française, les matériaux recueillis dans le cadre du programme Refmanpol (cf. note méthodologique) révèlent tout d'abord l'importance croissante acquise par les activités de chiffrage au sein de la police nationale : si le chiffre compte pour la police française, c'est d'abord parce qu'il occupe de plus en plus d'agents de l'institution policière tandis que le principe même des indicateurs de performance alimente la multiplication des données chiffrées (1). Ils montrent ensuite que l'utilisation d'indicateurs chiffrés tend à renforcer les traits centralisateurs de l'administration policière française : chaque échelon doit rendre des comptes à la strate supérieure (2). Cependant, cette logique de remontée d'informations suscite des réactions et comportements divers de la part des échelons subalternes, qui mettent en œuvre différentes stratégies d'ajustement pour gérer les demandes de plus en plus urgentes de la part de la hiérarchie (3). De plus, le contrôle induit par l'utilisation d'indicateurs et d'objectifs, bien qu'il soit ressenti comme pressant, reste partiel, tant les aménagements, bricolages, voire tricheries, permettent de contourner les exigences de l'échelon supérieur (4).

\section{Note méthodologique}

La recherche qui nourrit cet article repose sur un travail de comparaison franco-britannique ${ }^{7}$. Le cas français est étudié à travers l'analyse de 4 commissariats : 2 relevant de la Préfecture de Police de Paris (PP), 2 relevant de la Direction Départementale de la Sécurité Publique (DDSP) du Pas de Calais ; des entretiens ont par ailleurs été menés au niveau ministériel : à la Direction Centrale de la Sécurité Publique et à l'état-major de la Direction de la Sécurité de Proximité de l'Agglomération Parisienne (DSPAP). Pour la partie française, exploitée dans le cadre de cet article, nous avons réalisé 41 entretiens exploratoires (pour la plupart au niveau central) et 87 entretiens sur les terrains locaux ( 35 pour la préfecture de police, 52 pour la direction départementale du Pas de Calais). Une telle recherche comporte une difficulté méthodologique centrale : compte tenu du caractère délicat de la question, notamment en raison de sa politisation, il est difficile de parvenir à dépasser le discours convenu ou les formules toutes prêtes. Pour dépasser cette difficulté, nous avons d'une part multiplié les entretiens : nous avons réalisé une vingtaine d'entretiens sur chacun des sites, ce qui nous permet de croiser les discours et les récits. Nous avons d'autre part constitué, au fur et à mesure de l'avancée de la recherche, un réseau d'interlocuteurs privilégiés, que nous avons rencontrés plusieurs fois, ce qui nous a permis de réinterroger certains d'entre eux sur des enjeux apparus au cours d'autres entretiens. Nous avons également, ponctuellement, réalisé des observations de réunion. Pour traiter d'un sujet comme celui-ci, parvenir à entretenir une familiarité avec le terrain est nécessaire si l'on veut éviter le clivage souligné par G. Marx : "Much of the literature on controversial police topics breaks down into two categories: uncritical work by well informed insiders, and critical work by uninformed outsiders » (Marx, 1988).

7 Cette recherche, intitulée Refmanpol (Réformes managériales des polices), est financée par l'ANR (Agence nationale de la Recherche). 


\section{Le chiffrage : une activité en développement au sein de la police nationale}

L'usage des indicateurs chiffrés n'est pas nouveau dans la police, comme plus généralement dans les institutions pénales. Au cours du XIX ${ }^{\mathrm{e}}$ siècle, la productivité judiciaire de la police et de la gendarmerie fait partie du débat public et il s'agit pour ces deux institutions de produire les «bons » chiffres pour se légitimer et répondre aux critiques d'impéritie qui leur sont faites (Lopez, 2007). S'il n'y a donc pas de radicale nouveauté, l'usage des indicateurs chiffrés a pris une importance grandissante. En 2013, il existe ainsi 73 « indicateurs opérationnels du dialogue de gestion » pour la police nationale, répartis en 6 rubriques, correspondant aux différentes missions de la police (ordre public et protection de la souveraineté, sécurité et paix publiques, sécurité routière, etc.). À tous les niveaux de l'administration, l'utilisation de tableaux de bord est désormais monnaie courante. Ces tableaux de bord sont constitués de trois séries d'indicateurs : des indicateurs de situation (l'évolution des différents types de crimes et délits), des indicateurs d'activité (enquêtes criminelles, contrôles routiers, interventions de la police scientifique et technique, etc.) et des indicateurs de ressources disponibles (présence sur la voie publique, nombre de congés maladie, etc.). Ces indicateurs proviennent de deux sources principales :

- «l'état $4001 »^{8}$, qui désigne globalement la statistique judiciaire et couvre les grandes catégories d'infractions enregistrées par les services. Ce sont ici les chiffres « classiques » de la police, qui renvoient à l'évolution de la délinquance, mais aussi à l'activité judiciaire (et notamment au nombre d'affaires élucidées par les services de police);

- la Main courante informatisée ${ }^{9}$, qui mesure principalement l'activité de la police, y compris quand cela n'entraîne aucun résultat judiciaire : il peut s'agir de la présence sur la voie publique, du nombre d'interventions réalisées ou encore des congés maladies déposés. Les fonctionnaires de police doivent en effet répertorier de façon quotidienne toutes leurs interventions sur la voie publique et comptabiliser le temps consacré à telle ou telle activité au cours d'une vacation.

Des tableaux de bord sont construits avec ces données au niveau de chaque circonscription comme au niveau départemental (DDSP en grande couronne et en province, DTSP à Paris et petite couronne), ce qui permet une comparaison des résultats de chaque circonscription (en termes de situation, d'activité et de ressources).

À ces indicateurs formatés nationalement, il faut ajouter tous les tableaux de bord utilisés au sein des commissariats, généralement bricolés à partir de tableurs informatiques, qui permettent de mesurer l'activité des services de voie publique (par exemple en termes de nombre d'interpellations, de gardes à vue, de contraventions, etc.) ou l'activité des unités d'investigation (avec notamment un suivi des dossiers traités par fonctionnaire).

La multiplication de ces indicateurs chiffrés s'explique en partie par des transformations technologiques, qui favorisent une logique de numérisation, d'enregistrement automatique et de transmission immédiate des données chiffrées. Ces nouvelles technologies

\footnotetext{
${ }^{8}$ L'état 4001 est une statistique qui repose sur une nomenclature de 107 index correspondant à des natures d'infractions. Il permet de comptabiliser le nombre de crimes et délits constatés par la police ou la gendarmerie, ainsi que les faits élucidés, les gardes à vue et les mis en cause.

${ }^{9}$ Développée depuis le milieu des années 1990, la main courante informatisée est une base de données dans laquelle sont enregistrées les déclarations des usagers venus signaler des faits au commissariat sans qu'il y ait dépôt de plainte, ainsi que les comptes rendus d'intervention des équipages de voie publique.
} 
rendent possibles la production et la manipulation d'un grand nombre de données. Plus fondamentalement, le développement des tableaux de bord et indicateurs chiffrés est lié à la transformation managériale des organisations publiques, qui importent des normes, outils et discours venant du privé, ce qui se traduit par la diffusion d'outils et de discours associés à la notion de performance, avec une insistance sur l'obtention de « résultats » (Roché, 2008; Maillard et Savage, 2012).

Le changement de titre de la principale loi en la matière est emblématique de cette évolution : en 2009, la LOPSI (loi d'orientation et de programmation pour la sécurité intérieure) de 2002 a été rebaptisée LOPPSI (loi d'orientation et de programmation pour la performance de la sécurité intérieure). L'instruction du Ministère de l'intérieur du 28 juillet 2006, adressée aux directeurs centraux et aux préfets, est elle aussi illustrative d'une rhétorique qui place la notion de performance au cœur du discours adressé aux policiers : la référence à une « culture de la performance » y est explicite et il y est question de gestion par objectifs ( « en fixant des objectifs clairs et réalistes, après un dialogue au sein du service »), de gestion participative « favorisant la mobilisation du personnel et l'autonomisation », d'audit, pour « évaluer en permanence l'adéquation entre les objectifs et les moyens mis en œuvre », d'évaluation des « capacités individuelles ». Une telle logique encourage la production d'indicateurs de performance, rapportés à des objectifs chiffrés définis au préalable. La définition d'indicateurs devant conduire à un meilleur pilotage des services alimente à son tour la production d'autres indicateurs, permettant, suivant les interlocuteurs, de justifier les mauvais résultats obtenus, de souligner le peu de pertinence d'un indicateur d'activité qui ne prend pas en compte les moyens disponibles ou encore de montrer que tout a été mis en œuvre pour obtenir les meilleurs résultats (actes effectués, initiatives prises, etc.). Ainsi, le chiffre appelle le chiffre, tandis que logique de reporting (rendre compte de ce que l'on fait) et logique de pilotage par la performance apparaissent dans les faits étroitement liées.

Cette montée en puissance des indicateurs chiffrés s'est accompagnée de la définition de nouvelles responsabilités et de nouvelles tâches mais aussi de changements dans les modalités de formation, de rémunération et d'évaluation au sein de la police. Ainsi, la fixation d'objectifs explicites et la construction d'indicateurs de performance pour évaluer l'efficacité et l'efficience des unités sont aujourd'hui un élément clé du travail des agents d'encadrement, dans la police comme dans la gendarmerie d'ailleurs. Dans le cadre de la LOLF (loi organique relative aux lois de finances), le directeur général de la Police nationale, comme chaque directeur de programme, doit élaborer un plan annuel de performance, en définissant des objectifs et des indicateurs de performance. Par ailleurs, les processus d'évaluation des commissaires ont changé en 2004 : ils prennent aujourd'hui compte des méthodes d'évaluation du secteur privé, de « l'atteinte des résultats au regard d'objectifs préalablement déterminés en fonction des moyens mis à disposition ${ }^{10}$. Dans cette logique, depuis 2010, les chefs de service, à la tête des commissariats, doivent élaborer des «projets de performance individuels » $(\mathrm{PPI})^{11}$, qui peuvent leur permettre d'obtenir des primes s'ils atteignent le ou les objectifs qu'ils se sont fixés avec leur hiérarchie. À l'échelle de l'ensemble de l'organisation policière, des modes de rémunération liés à la performance ont été mis en place à partir de 2004, avec initialement un budget de 5 millions d'euros consacré

\footnotetext{
10 Protocole d'accord sur la réforme des corps et carrière, 17 juin 2004.

11 Mis en place par décret du 21 septembre 2010.
} 
aux primes individuelles et collectives. Ce budget a progressivement augmenté, passant de 10 millions en 2005 à 25 millions à partir de 2008 pour la prime de résultat exceptionnel ${ }^{12}$.

L'importance croissante des indicateurs de performance est attestée par l'existence de nouvelles spécialisations au sein de la police, certaines personnes étant spécifiquement en charge de la collecte des informations et du traitement des statistiques. À Paris, les commissaires centraux d'arrondissements sont secondés depuis le début des années 2000 par un Bureau de Coordination Opérationnelle $(\mathrm{BCO})$ directement rattaché à ces derniers. L'une de leurs missions est la compilation et l'enregistrement des données statistiques, ainsi que la réponse aux demandes d'information formulées par l'échelon hiérarchique supérieur, à savoir le district. Au sein de l'état-major de la DSPAP, un bureau est en charge du suivi des indicateurs chiffrés, le bureau d'analyse des statistiques, qui comprend une quinzaine de personnes. Dans le département du Pas-de-Calais, une douzaine d'agents procèdent au recueil et au traitement des statistiques au sein de la DDSP, en plus des responsables des tableaux de bord dans chaque commissariat : c'est la tâche spécifique du BASSO (Bureau d'Analyse des Statistiques et des Synthèses Opérationnelles), qui comprend 5 personnes, et du contrôleur de gestion mais de nombreux autres services ont également des activités statistiques : le BACO (Bureau d'Aide au Commandement) mais aussi le « bureau du partenariat », le bureau du personnel, le « coordinateur tir », le BOPSR (Bureau d'Ordre Public et Sécurité Routière).

\section{Indicateurs chiffrés et renforcement de la centralisation}

Bien que l'instruction ministérielle de 2006 évoquée ci-dessus insiste sur le processus participatif de définition des objectifs, une telle multiplication des indicateurs chiffrés tend à renforcer la structure hiérarchique de la police nationale.

La police française se structure autour de trois nœuds de relations hiérarchiques : entre les niveaux national et départemental, entre les niveaux départemental et local et au sein des commissariats. Dans l'agglomération parisienne, la situation diffère du fait de l'organisation spécifique de la Préfecture de Police mais reproduit ces trois nœuds, à ceci près que le premier nœud associe niveau «central » (cabinet du préfet et DSPAP) et niveau départemental (Directions Territoriales de Paris et des trois départements de la petite couronne). À ces trois niveaux, les nouvelles techniques de management ont tendance à renforcer les traits centralisés du système français.

\subsection{Du ministère au commissariat : une centralisation renforcée}

La définition des priorités s'exerce d'abord au niveau ministériel. Les objectifs définis par la Direction centrale de la sécurité publique, en accord avec le ministre et la direction générale, sont transmis au niveau départemental (DDSP), qui doit les décliner en tenant

${ }^{12}$ D'après le rapport de la Cour des Comptes (2013), « cette prime a été perçue par 44438 fonctionnaires de police en 2010 (au lieu de 28000 en 2005), soit $30 \%$ des effectifs du programme, pour un montant moyen de $562 €$ (au lieu de $357 €$ en 2005) » (p. 123). Le montant total des primes est à rapporter au montant total des dépenses de rémunérations dans la police nationale : 4,5 milliards d'euros en 2006, 4,8 milliards en 2010, 4,9 milliards en 2011, hors cotisations et prestations sociales. 
compte des conditions locales. Ces objectifs sont d'un côté des « objectifs prioritaires de lutte contre la délinquance », qui prennent la forme de baisses attendues des différents types de crimes et délits (baisse de $\mathrm{x} \%$ des atteintes volontaires à l'intégrité physique (AVIP) par exemple) et de hausses attendues des taux d'élucidation par catégorie d'infraction (hausse de y $\%$ pour les AVIP par exemple). Certaines infractions peuvent par ailleurs faire l'objet d'une attention particulière en raison de leur fréquence ${ }^{13}$. Ce sont d'un autre côté des objectifs dits de «pilotage de l'activité des services » (taux de transport sur les vols à la roulotte, nombre d'agents sur la voie publique à un instant t, par exemple) ou encore des objectifs de «pilotage des relations police/population» (« réunions et contacts avec les usagers et acteurs de la vie publique » par exemple).

Une telle procédure de déclinaison d'objectifs nationaux génère un processus complexe, dans lequel les directeurs départementaux doivent prendre en compte les conditions locales et les priorités nationales, mais elle ne donne pas vraiment lieu à une «négociation locale » . Les DDSP veillent plutôt à répondre aux attentes de leur hiérarchie, qui les évalue ${ }^{14}$. Un autre processus tend à renforcer la centralisation : la remontée statistique régulière vers le ministère, à un rythme qui a été mensuel, puis hebdomadaire, sans compter la réunion hebdomadaire avec le Préfet et les demandes d'informations thématiques :

«À Paris, ils font également des études sur des thématiques, sur les accidents de la route, sur les vols de téléphones portables, sur les agressions des enfants dans les écoles. C'est normal. Paris nous sollicite "Dans votre département, comment ça fonctionne? Qu'est-ce que vous avez comme chiffres? Faites-moi des études”. Là, c'est tout informatisé, ils ont directement accès à nos chiffres. Quand ils nous sollicitent, ils veulent un peu une appréciation littérale, comment sont les choses » (état-major, direction départementale, octobre 2012).

La remontée d'information statistique n'est pas un processus neutre : elle comporte clairement une dimension hiérarchique. Faire remonter ses chiffres, c'est une façon de rendre des comptes sur son action, c'est potentiellement se faire questionner sur son action. Ceci crée une pression généralisée sur les acteurs locaux, d'autant plus lorsque les mauvais élèves peuvent être pointés du doigt ${ }^{15}$. La demande de statistiques peut alors effectivement agir comme un outil de gouvernement, l'exigence de remontées mensuelles ou hebdomadaires étant vue comme un signe de contrôle, tandis que l'existence d'un système de récompense, via le système des primes collectives au niveau départemental, installe l'idée qu'il est bon d'atteindre les objectifs fixés. C'est ainsi qu'interrogé sur ce qui se passe si les objectifs départementaux ne sont pas atteints, un chef d'état-major commence par répondre « rien!» avant de préciser :

- "Si les objectifs départementaux ne sont pas atteints, il y a une sanction, si je peux utiliser cette expression, c'est qu'il y a des primes qui sont données par l'État, des

\footnotetext{
${ }^{13}$ Les cambriolages étaient par exemple l'une des principales préoccupations pendant le travail de terrain. 14 II convient cependant de rappeler que les DDSP ont été évalués pendant un temps (jusqu'en 2011) au niveau local par le préfet.

${ }^{15}$ Nicolas Sarkozy, ministre de de l'intérieur entre 2002 et 2004 puis de 2005 à 2007, avait ainsi mis en place un "Sarkomètre », consistant à convoquer à son ministère les Préfets, DDSP et Commandants de groupements de gendarmerie des cinq départements ayant eu les pires résultats et des cinq ayant eu les meilleurs résultats. Ceci rappelle le «dramaturgic appeal » de certains discours évoqués par Manning à propos des polices britanniques et américaines (2001).
} 
primes collectives : il y a des départements qui sont sélectionnés quand ils ont des bons résultats, ils ont des primes. Nous cette année on a eu la prime collective. Donc c'est aussi un critère de prise en compte des résultats. (...) Celui qui a eu la prime collective l'année d'avant, le Directeur Départemental il a envie d'avoir des bons résultats, pas pour avoir la prime collective, mais pour éviter de ne pas l'avoir. » (décembre 2012). Les relations entre DDSP et commissariats sont affectées de la même manière. La multiplication des systèmes de suivi, largement informatisés, procure de nouvelles sources d'information au niveau départemental. Cela permet un suivi quotidien de l'activité des commissariats par les chefs d'état-major, qui peuvent appeler les commissaires quand ils repèrent des chiffres « anormaux » ou des évolutions négatives. Un chef d'état-major nous confiait ainsi :

"Tous les matins, on m'amène les chiffres, l'analyse de la délinquance, circonscription par circonscription [de fait les tableaux sont sur son bureau]. On appelle les circos, si par exemple y'a des cambriolages ou des vols roulotte, je fais faire des études par le BACO pour voir dans quel secteur ça augmente et ensuite on transmet les études aux circos.

- Q : Vous ne les appelez pas au jour le jour quand même?

- Jour le jour, la réponse est non mais deux fois par semaine oui. » (décembre 2012).

Cette situation se reproduit avec une acuité plus grande encore dans le ressort de la Préfecture de Police, notamment depuis la mise en place de la police d'agglomération en $2009^{16}$, qui a placé sous son autorité les trois départements de la petite couronne. Le fonctionnement de la PP se caractérise par une très forte demande de remontées de statistiques et d'informations à la direction et au cabinet du Préfet de Police : la « centrifugeuse parisienne », pour reprendre l'expression d'un commissaire rencontré au cours de cette recherche, impose un certain nombre de $«$ plans $»^{17}$, d'objectifs et de priorités aux circonscriptions locales, quitte parfois à étendre à la banlieue des problématiques qui concernent avant tout Paris. Les remontées de chiffres correspondent ainsi à une volonté de contrôler la mise en œuvre de ces objectifs : demander à une circonscription le nombre de vendeurs à la sauvette ou de prostituées interpellées revient à vérifier que cette dernière met bien en œuvre cette priorité fixée par le Préfet.

Tout ceci a contribué à faire évoluer les commissaires d'une position relativement autonome, dans laquelle ils avaient peu de comptes à rendre, à une double responsabilisation : en interne, ils doivent rendre des comptes à leur direction, non seulement en termes de résultats chiffrés, mais aussi sur des questions organisationnelles telles que la capacité de traiter avec les syndicats; vis-à-vis de leur environnement politico-social, ils doivent rendre des comptes sur les statistiques de la délinquance et être en mesure de communiquer avec les acteurs locaux :

«Moi en gros j'ai trois directeurs ou quatre directeurs. J'ai le préfet, le procureur, mon directeur administratif et le maire. Ça c'est mes quatre chefs. C'est là que je prends mes instructions, enfin en tout cas mes orientations et c'est à moi après de faire le mélange avec tout ça. En outre, le système de récompense incite à privilégier les objectifs du Ministère plutôt que les priorités locales. » (commissaire, Préfecture de police, février 2012)

Dans le cas français, la centralisation est d'autant plus confortée par la multiplication des

\footnotetext{
${ }^{16}$ Décret $n^{\circ} 2009-898$ du 24/07/2009

${ }^{17}$ La politique de la PP sous Michel Gaudin (2007-2012) s'est en effet caractérisée par un certain nombre de « plans », pour lutter contre les bandes, les cambriolages, la drogue...
} 
tableaux de bord et des indicateurs chiffrés qu'il n'y a pas de dynamique participative. Seuls les projets de performance individuels des commissaires sont définis par les intéressés. Ce phénomène de centralisation n'est d'ailleurs pas l'apanage des administrations fortement hiérarchisées comme la police. Frédéric Pierru (2013,p. 204) constate ainsi, pour le cas des hôpitaux, que les réformes managériales de quantification et de suivi/contrôle des activités ont induit « de la verticalité et de la hiérarchie là où prévalaient des organisations réticulaires et horizontales ». Dans le cas de la police, cette logique de remontée d'informations et de contrôle des subordonnés se retrouve également au sein des commissariats.

\subsection{Un renforcement de la redevabilité interne aux commissariats}

Au sein des commissariats, les indicateurs chiffrés peuvent être utilisés comme un outil de mesure, de suivi et, parfois, de contrôle des activités. Dans beaucoup de commissariats, les chefs de service ont à leur disposition des unités en charge de la collecte des données et de l'analyse des statistiques. Ainsi, dans chaque commissariat relevant de la Préfecture de police (Paris), il existe un Bureau de Coordination Opérationnelle (cf. supra). Par ailleurs, les tableaux de bord et outils informatiques évoqués ci-dessus sont alimentés quotidiennement par les policiers de terrain, qui font ainsi remonter des informations vers leurs supérieurs.

Cette logique de reporting généralisé s'avère être une nouveauté relative pour les gradés (brigadiers, brigadiers-chefs et majors). Ces derniers doivent désormais rendre régulièrement des comptes chiffrés sur leur action en établissant des tableaux de bord qu'ils font remonter sur une base régulière, notamment pour les unités de voie publique. À certaines périodes, cette logique de remontée a pu être accompagnée d'objectifs quantifiés fixés aux services :

«Des statistiques étaient tenues sur l'ordinateur, des tableaux avec le nombre d'interpellations par groupe et il y avait des tableaux où c'était notre nom. Tous les noms des collègues étaient là, le nombre de jours travaillés. J'étais la première à être barjot parce que j'ai dit « Je ne travaille pas à l'Insee moi ». Le nombre d'interpellations par nombre de jours travaillés, ça vous faisait un ratio » (gradée, voie publique, Préfecture de police, novembre 2012).

«Nous on était tenu de relayer les informations de la direction et on avait des objectifs à se fixer, les commandants et nous. Et puis c'étaient des objectifs de tout : de contraventions, d'accidents, de mises à disposition, et en fin de mois, on avait un compte rendu à faire : faits constatés, ambiance de la brigade, les malades, les présents, les interpellations, le nombre de contrôles routiers par collègue. Tout était détaillé sur papier. On rendait le rapport au commandant et on le regardait avec lui. Lui il en faisait un, toutes brigades confondues, qu'il transmettait à la direction » (major, voie publique, Préfecture de police, octobre 2012). Il est attendu de cette logique de remontée d'information systématique qu'elle renforce l'implication des cadres intermédiaires dans le suivi des équipes opérationnelles. Cela se produit dans un contexte de redéfinition de la hiérarchie interne, avec un nouveau rôle donné aux officiers. En effet, alors qu'ils ont pendant longtemps assuré principalement des fonctions d'inspecteurs, avec des activités d'enquête, ils doivent aujourd'hui assumer de plus en plus de fonctions d'encadrement :

"Chaque unité doit rédiger une fiche synthétique de son activité, par quinzaine pour qu'on fasse un bilan global en fin de mois. Pourquoi on fait un bilan synthétique au milieu du mois? Pour pouvoir rectifier. Pour ne pas s'apercevoir en fin de mois qu'il y a une brigade ou une unité quelconque qui a eu un manque d'activité certain. C'est pour pas 
se réveiller trop tard. (...). Chaque unité doit nous donner son nombre d'interpellations et son nombre de PV. Non pas en se disant "y a un quota" y a pas de chiffres, c'est juste pour s'assurer qu'il y a une certaine activité. L'activité des interpellations, c'est eux qui nous les retracent. Qui dit interpellation dit PV d'interpellation donc on peut vérifier leurs dires » (officier, voie publique, Préfecture de police, novembre 2012).

Là encore, ce résultat est convergent avec ce qui est constaté à propos des « réformes managériales de l'hôpital », caractérisées par « la multiplication des hiérarchies intermédiaires entre la chefferie de l'établissement et les activités professionnelles proprement dites » (Pierru, 2013, p. 205). Les indicateurs semblent ainsi consolider l'intégration organisationnelle; c'est d'ailleurs ce que soulignent A. Purenne et J. Aust (2010) dans leur propre travail sur la police. De fait, la base d'un chaînage hiérarchique solide est le caractère pyramidal de la circulation de l'information, qui doit remonter du bas vers le haut pour alimenter les décisions. La systématicité des remontées d'information, sous la forme de tableaux divers, s'inscrit parfaitement dans une telle logique. Il y a ici l'ambition de rationaliser l'organisation policière, en la rendant plus transparente à elle-même et en s'assurant du suivi de l'activité par la hiérarchie.

Pour autant, les remontées d'information sont variables en fonction des commissariats : dans l'un des commissariats de la PP, des tableaux sont ainsi transmis depuis plusieurs années à la hiérarchie intermédiaire (officiers) toutes les deux semaines et à la hiérarchie supérieure (commissaires) chaque mois tandis que dans l'un des commissariats du Pasde-Calais, ce n'est que depuis fin 2013 que tous les services de voie publique opèrent des remontées informatisées mensuelles à leur hiérarchie directe. Par ailleurs, ce processus est source de tensions internes : la récurrence des remontées d'informations peut susciter une forme d'insatisfaction interne, qui peut même produire des stratégies de contournement.

\section{Critiques de la logique bureaucratique}

Le système centralisé alimente donc des demandes multiples de la part des autorités centrales, qui sont dans une position de recherche d'information : elles peuvent demander de l'information pour élaborer les plans, préparer des décisions ou encore suivre l'exécution de certaines mesures. Le caractère répété des demandes, en particulier au sein de la Préfecture de Police, suscite une forme de mécontentement des agents face à une activité jugée chronophage, répétitive et inutile (3.1), voire des comportements non-coopératifs, notamment quand ils se retrouvent confrontés à une commande à laquelle ils ne peuvent pas répondre de façon satisfaisante (3.2). « Le chiffre » est ici assimilé à une dérive bureaucratique, qui alourdit excessivement le travail.

\subsection{Urgences et demandes répétées : les épreuves de la centralisation}

Si l'information remonte, ce n'est pas sans susciter certaines réserves de la part des acteurs chargés de la collecter. Si les réactions sont susceptibles de varier, en fonction du degré de spécialisation, du type d'activités et du positionnement hiérarchique, c'est un écho critique qui ressort des entretiens. Les agents chargés de la remontée d'information insistent sur le caractère excessivement urgent, répétitif, parfois infondé, des demandes qui leur sont faites. Pour ce qui est du caractère d'urgence ressenti pour certaines des demandes, celles formulées par la direction centrale de la sécurité publique ou la direction 
de la sécurité publique de l'agglomération parisienne peuvent mettre sous pression les agents chargés de leur répondre :

"C'est toujours pour hier et c'est toujours où il faut aller rechercher des milliards d'archives... Enfin, des tableaux, des machins, des choses qui prennent du temps et qui au bout du compte bloquent les gens dans les services, alors même que c'est exactement ce qu'au niveau central on ne souhaite pas. C'est-à-dire qu'on souhaite que les gens soient dehors mais, en fait, on multiplie tellement les demandes qu'on est obligé de gonfler nos états-majors » (commissaire, Préfecture de police, juillet 2012).

"Ce qui est contraignant pour nous, c'est le délai. C'est des délais qui sont extrêmement restreints. C'est-à-dire que dans les études, on n'a pas vraiment le temps de se mettre dans un coin et de réfléchir tout de suite. Je veux dire qu'en général, le délai il est de deux heures des fois, des fois moins, des fois c'est 24 heures » (état-major, direction territoriale, Préfecture de police, juillet 2012).

Par ailleurs, ces transmissions d'information se font souvent sous des formes proches à des destinataires différents et selon des temporalités légèrement décalées. Les agents sont de ce fait amenés à produire un travail répétitif sans nécessairement pouvoir profiter d'économies d'échelles liées à cette répétition, celle-ci n'étant jamais absolue. C'est ce que dénoncent certains agents fatigués de produire une information pour différentes directions :

"On a beaucoup de remontées ponctuelles sur des sujets précis... Mais le problème c'est qu'on nous demande 4 ou 5 fois la même chose, pour 5 demandeurs différents (état-major, direction territoriale, Préfecture de police, avril 2012).

Enfin, la fréquence et la variété des demandes venant de l'échelon central sont génératrices d'une certaine lassitude pour les fonctionnaires qui doivent y répondre. L'accumulation des demandes, leur caractère parfois «politique » et déconnecté des réalités de terrain suscite des réactions négatives :

"Ça c'est un côté qui est vraiment contraignant quoi. Ça c'est un effet un peu pervers pour nous de la technostructure DSPAP et puis le fait qu'on soit rattaché à la PP, c'est qu'il y a des commandes incessantes sur un état, sur tel sujet, dans tel domaine "Pour midi, il nous faut le nombre de vendeurs à la sauvette que vous avez arrêtés depuis ce début de l'année" c'est tous sujets, ça part dans tous les sens» (officier, Préfecture de police, octobre 2012).

"On s'est aperçu qu'on avait pas mal de demandes dans l'année qui a précédé les (élections)... de toute façon, on savait qu'on allait en baver. Les présidentielles arrivent, ça retombe sans qu'il y ait un outil qui se mette en place. Les demandes repartent...(...) Il y avait vraiment des remontées pour tout » (officier, bureau d'analyse statistique, direction départementale, octobre 2012).

Les demandes étant pensées au niveau central, elles ne font pas nécessairement sens dans les territoires auxquels elles sont adressées, ce qui ne manque pas de produire une forme d'insatisfaction devant le caractère excessivement uniformisant de la demande pour ceux qui sont sommés de faire remonter l'information alors qu'ils ne sont pas concernés par les problèmes :

"Et puis il y a cette tendance à vouloir unilatéraliser les problèmes. La prostitution, il n'y en a pas qu'à Paris, à X, je ne dis pas qu'il n'y en a pas, mais j'ai trois Roumaines qui tapinent place $Y$, point barre. Ce n'est pas un phénomène [important] mais il y a une étude sur la prostitution qui est menée! (...) Toute la plaque d'agglomération va devoir rédiger une espèce de compte rendu. Alors, c'est des tableaux tout prêts, il faut mettre le nombre 
d'arrestations, le nombre de procédures initiées, le nombre de proxénètes interpellés. Tout le monde met zéro. Ce n'est pas l'échelle parisienne donc c'est un non sujet. Ils ne savent plus faire la part des choses. » (commissaire, Préfecture de police, juillet 2012). Ce processus de remontée de l'information est complexifié par la multiplication des échelons. La police est en effet une organisation caractérisée par une abondance de niveaux hiérarchiques qui, chacun à leur tour, transmettent les demandes de l'échelon supérieur :

"Il y a quand même, comme je l'ai dit, les étapes hiérarchiques. Le cabinet du préfet appelle la DSPAP, qui va appeler les directions. Les directions vont nous appeler, donc quand même, malgré tout, une espèce de lourdeur à l'aller et au retour, avec des délais qu'on contraint du coup, parce qu'on veut quand même répondre à l'avance. Donc, chacun prend une bonne marge et du coup nous, pression maximale à l'arrivée, parce que c'est nous qui avons l'information » (chef de circonscription, Préfecture de police, juin 2012).

En définitive, la multiplication des niveaux allonge la temporalité du processus et a pour effet d'augmenter les tensions entre chacun des niveaux. La répétition des demandes place les services chargés de la collecte des données dans une position malaisée, entre les impératifs des directions centrales et les réticences des agents de terrain. Un responsable de bureau nous expliquait :

"En plus quand vous faites une demande chiffrée et que vous demandez aux gens d'expliquer le pourquoi de l'évolution, il y a des gens qui ont d'autres choses à faire. On se met à leur place. On a travaillé en commissariat et quand les demandes tombent, la réaction c'est toujours la même "Ils ont vraiment rien à foutre à la DDSP". On se dit "Tiens, le gars il s'est réveillé ce matin et il se demande comment il va pouvoir nous embêter aujourd' hui", alors que la DDSP ne fait que relayer une demande » (officier, responsable de bureau d'analyse statistique, direction départementale de sécurité publique, octobre 2012).

\subsection{Le maintien d'une forme d'opacité organisationnelle}

Les demandes ponctuelles, et donc non anticipées par le destinataire, font que le niveau subalterne, censé alimenter les bases de données, peut produire de l'information erronée. Pressé de répondre, sans avoir des moyens de collecte suffisants et ne croyant pas à la légitimité de la demande, il peut être conduit à fournir une information faussée, qui lui permet de s'arranger formellement avec les exigences de la hiérarchie. Certaines citations soulèvent ainsi la question de la qualité et de la fiabilité de l'information produite :

"Pour les interrogations intelligentes, on va rechercher le chiffre; les interrogations stupides du type "Patron, l'état-major il demande le nombre de pffff'. J'appelle mon secrétariat et je dis "Donnez-moi un chiffre entre 0 et 100". - Je vous assure! - "37". Je vous assure que dans $90 \%$ des cas c'est très bien, ça ne revient jamais. Si jamais ça revient : "37 ?". Pour le coup, je vérifie vraiment. Très honnêtement, il y a vraiment des questions à la c. où, je vous dis, mon secrétaire me demande, j'appelle mon chauffeur et je dis “Donnez-moi un chiffre entre 0 et 100" (commissaire, Préfecture de police, mai 2013). "À un moment donné, on n'a pas les études, on n'a pas l'outil statistique. On nous demande le nombre de mecs conduits dans les $U M J^{18}$ depuis 2 ans... Mais on ne les a pas, ces chiffres. On passe notre vie à chercher des données... On a des commandes qui

\footnotetext{
18 Unités médico-judiciaires.
} 
arrivent, qui sont de vraies galères... Du coup, on donne des chiffres approximatifs, voire même on les invente » (commissaire, Préfecture de police, juin 2013).

Le système est donc loin d'être complètement rationalisé, avec une information circulant de façon parfaitement fluide. Les demandes d'information, à tous les niveaux, font l'objet de retraductions, de réappropriations mais aussi, parfois, de rejets. Le mélange de distance critique vis-à-vis des outils mis en place par l'institution et d'utilisation accrue des indicateurs chiffrés a d'ailleurs pour effet de les démultiplier : en effet, un certain nombre d'acteurs rencontrés ont mis en place, de façon plus ou moins informelle, des outils de mesure «bricolés » en interne, soit pour piloter plus efficacement l'activité d'un service, les outils fournis par la hiérarchie (MCI, cartographie criminelle...) étant jugés peu fiables car trop lents ou mal remplis, soit pour se prémunir contre d'éventuelles critiques venues de la hiérarchie, les deux motifs n'étant évidemment pas mutuellement exclusifs. Ainsi, de nombreux responsables d'unités et chefs de service ont construit leurs propres tableaux :

"J'ai fait des outils à moi que le major qui travaille avec moi remplit, ou moi quand il n'est pas là. Suivi de l'activité quotidienne. (...) Ça va me servir juste à me justifier. Lorsqu'on va me faire des reproches que l'activité est en baisse, si par exemple on a eu 16 personnes qui ont fait de la garde dans des hôpitaux, qui ont fait du statique sur des fêtes, sur des matches de foot. Tout ça additionné, c'est du temps qu'on ne peut pas donner ailleurs, donc on ne peut pas faire de contrôle routier, on ne peut pas interpeller, on ne peut pas sécuriser... parce qu'on est ailleurs. C'est plus un justificatif pour expliquer pourquoi les résultats sont plus ou moins bons et un justificatif qu'on peut prouver » (officier, voie publique, Préfecture de police, octobre 2012).

"On a créé notre tableau Excel pour pas qu'on nous réclame des dossiers qu'on n'a jamais eu...du jour, qu'ils ont créé un dossier Excel, on s'en est aussi créé un dans la brigade » (brigadier, circonscription de sécurité publique, novembre 2013)

Autrement dit, l'observation du mode de production de l'information révèle le caractère centrifuge de l'organisation policière, faite de segments administratifs autonomes et pour parties concurrents. Surtout, elle met en évidence les tensions entre le haut et le bas, ainsi que l'écart entre l'ambition synoptique des autorités centrales et les capacités de production d'information du système, comme le montre aussi l'usage des indicateurs dans l'assignation des objectifs et le suivi des unités.

\section{Piloter par les chiffres : recherche de conformité et tensions organisationnelles}

On l'a vu, les chiffres exercent une pression sur les différents niveaux hiérarchiques par les remontées d'informations qu'ils génèrent. Ils sont également une source de pression pour les agents du fait de $1^{\prime}$ attente de résultats liée à ces indicateurs chiffrés. Plusieurs entretiens confirment les critiques émises à l'encontre de la « culture du résultat » en ce qu'elle serait à l'origine de trucages des statistiques. La recherche de bons chiffres conduit ainsi à un certain nombre d'arrangements destinés à satisfaire les attentes de la hiérarchie (4.1). Ces mêmes chiffres peuvent être source de désaccords entre les différentes strates sur la pertinence de certains des objectifs demandés. L'étude révèle ainsi des clivages organisationnels se cristallisant autour de cette question des indicateurs chiffrés (4.2). 


\subsection{Jouer avec les chiffres : un arrangement implicite}

Les chiffres de la délinquance sont devenus un élément central dans la façon dont est politiquement évaluée l'efficacité des services de police (Monjardet, 2006; Mucchielli, 2008). Mais plus l'information policière est politisée (sur des indicateurs tels que l'évolution de la criminalité ou le taux d'élucidation), plus elle est sujette à manipulation. En effet, la police et les acteurs politiques ont intérêt à produire des informations démontrant une baisse de la délinquance et une augmentation du taux d'élucidation; or, ces informations sont produites par la police elle-même, qui peut donc « tordre » les chiffres à son avantage. La manipulation des chiffres est de fait relativement courante (Matelly et Mouhanna, 2007). Ces pratiques françaises font d'ailleurs écho à celles observées ailleurs : sous-enregistrement des faits, qui peut passer par des systèmes d'enregistrement plus contraignants ou par des changements dans la nature des faits pris en compte (Patrick, 2011; Eterno et Silverman, 2012); collusion, entre les agents et les suspects, visant à faire avouer un grand nombre d'infractions, en échange de faveurs telles que des réductions de peine, la visite des conjoints, la fourniture de drogues ou d'alcool (Patrick, 2011).

Les demandes de « bons chiffres » par la hiérarchie peuvent être insistantes, et conduire les commissaires à des stratégies de mise en conformité où ils utilisent les marges offertes par le système. Deux chefs de service rencontrés dans le cadre de la recherche nous ont ainsi rapporté des exemples précis de pression exercée par leur supérieur hiérarchique pour améliorer des résultats en matière d'évolution de la délinquance. Sans que cela soit formulé explicitement, ils doivent trouver des moyens de faire baisser l'évolution de la délinquance dans leur circonscription, en étant imaginatifs. Face à une telle exigence, ils dégagent une solution, qui fait l'objet d'un accord implicite avec leur hiérarchie : retarder l'enregistrement des faits de délinquance, pour maintenir des bons chiffres. Cette pratique est celle du « lissage » (mensuel) des crimes et délits mais aussi des cas résolus.

"Quand il y a de très très très très mauvais chiffres, (votre chef) vous engueule pas, il demande des explications. 'Comment ça se fait que...'. En fait, il faut comprendre qu'il est en train de vous engueuler parce qu'il n'est pas content, mais il vous appelle. Moi, il me le faisait au tout début. (...) Il fait ça pendant trois-quatre jours. Vous comprenez qu'il vous met la pression et qu'il faut vous débrouiller pour que les voyants soient plus au vert. Alors, il y a plein de moyens différents, qui sont plus ou moins tolérés, plus ou moins institutionnalisés. Donc, au bout de six mois, vous comprenez que si vous ne rendez. pas une copie à peu près correcte en termes de voyants d'activité, vous avez tout le temps des coups de fil où on vous emmerde. Vous affichez des bons résultats et puis à côté, vous faites bien votre boulot. (...). Vous pouvez afficher des bons résultats qui sont pas truqués, parce que c'est pas du truquage, qui sont lissés » (commissaire, mai 2012).

"Après quelques mois, mon directeur, que j’apprécie beaucoup par ailleurs, me dit : “Ouais, tu me fais ch.(sic). On est là pour faire de la politique, on n'est pas là pour faire de la police". Mais je n'étais pas d'accord là-dessus et donc c'est vrai que la première année où je suis arrivé, les stats ont fortement augmenté parce que peut-être mon prédécesseur ne faisait pas comme ça et donc il y a un moment où ils ont commencé à vouloir me faire passer le truc à la trappe parce que j'ai bien évidemment refusé. Donc on a négocié si je puis dire, donc j'ai fait un peu de cavalerie, c'est-à-dire que j'enregistrais tout mais parfois avec un peu de décalage dans les mois. J'en mettais de côté et puis ils 
m'en laissaient enregistrer de temps en temps. Ce qui fait que les stats la première année ne voulaient absolument rien dire » (commissaire, février 2012).

Ces adaptations peuvent ensuite se répercuter le long de la chaîne hiérarchique. Le chef d'une unité d'investigation d'un commissariat nous a ainsi expliqué que son unité a pendant un temps gardé en réserve des cas faciles à résoudre afin de les « sortir » quand les statistiques mensuelles s'annonçaient plutôt mauvaises, ce qui est par ailleurs congruent avec leur souci d'avoir des « beaux dossiers » de façon régulière :

"On s'en gardait quelques-uns de côté pour les temps durs. On ne faisait même pas ça pour lisser les chiffres. Nous c'était plus dans le but de ne pas rester 3 mois sans faire d'affaire. Et puis, [pour ne pas] faire que de vaines recherches, ou si le travail ne nous plaît pas, on se garde les beaux dossiers, pour avoir toujours une activité intéressante. C'est plus eux [les supérieurs hiérarchiques] qui étaient dans le lissage » (novembre 2013).

Les remontées chiffrées régulières ont donc pour effet d'augmenter l'emprise et le contrôle hiérarchique : les chiffres remontent, sont suivis sur une base mensuelle et peuvent se traduire par des demandes plus ou moins clairement formulées d'amélioration des résultats. En même temps, les différents exemples rapportés ici témoignent du fait que des marges de manœuvre sont laissées aux agents : ces derniers atteignent les objectifs, mais en privilégiant les moyens sur les fins, ou encore en jouant sur l'enregistrement, comme dans le cas du codage des malades dans la T2A (tarification à l'activité) ${ }^{19}$. Cette logique de manipulation des chiffres, tout comme les données « fantaisistes » évoquées plus haut, montre le caractère paradoxal de la centralisation : les arrangements avec les chiffres résultent d'une injonction centrale, qui vise l'atteinte d'objectifs, mais ces arrangements procèdent d'une myriade de pratiques locales développées par les services de police. De ce point de vue, la police est à la fois incontestablement centralisée (il faut se conformer à des objectifs énoncés centralement) et profondément fragmentée (chacun des niveaux disposant d'une marge de manœuvre dans l'enregistrement et la présentation des données). Cette logique de résultats génère également un certain nombre de tensions dans les rapports hiérarchiques, les échelons supérieurs étant suspectés d'être uniquement rivés sur l'atteinte des objectifs chiffrés.

\subsection{L'usage des indicateurs comme source de clivages organisationnels}

À n'en pas douter, la pression sur les chiffres accroît l'écart entre l'intérieur (avec ses petites manœuvres) et l'extérieur (la communication sur des résultats positifs) de l'institution policière. Elle accroît également la distance entre les niveaux hiérarchiques. L'usage des indicateurs chiffrés participe ainsi des tensions internes à l'organisation policière.

Si l'on a insisté plus haut sur le fait que les ordres descendaient en cascade, cette logique est loin d'être absolue. À l'intérieur des commissariats, les demandes ne sont pas retraduites de façon transitive, créant de ce fait un certain nombre de décalages, voire d'incompréhensions. Si les officiers sont la plupart du temps fidèles à leurs supérieurs, ils adoptent parfois une position assez distante, voire une stratégie de contestation. Ainsi, dans certains commissariats, l'encadrement intermédiaire va implicitement à l'encontre

${ }^{19}$ F. Pierru (2013) a recours à une formule bien adaptée au cas de l'hôpital : la logique est de « soigner les indicateurs plutôt que les malades ». 
des objectifs de «hausse de rendement » fixés par les commissaires : certains officiers diffusent ainsi le message selon lequel il vaut mieux « ne pas trop en faire », pour ne pas qu'on leur en demande « encore plus » ou indiquent à leur hiérarchie qu'il est impossible de fournir de meilleurs résultats; ils prêtent par ailleurs peu d'attention aux tableaux qui remontent des unités :

«On peut penser que la direction en veut toujours plus. Le problème c'est que, toujours plus, on va pouvoir le faire, peut-être pendant un certain temps mais à un moment donné... Moi, c'est ce que je défends à chaque fois. Ce que je veux, c'est que les gens soient présents au maximum dehors, qu'ils occupent le terrain, qu'ils ouvrent leurs yeux, leurs oreilles, et qu'ils travaillent tout simplement. Mais on n'est pas une société privée, on ne peut pas dire qu'on donne un chiffre d'affaires à faire. Ce n'est pas possible. Ce n'est pas palpable ce que l'on fait. Je pense que la meilleure façon de faire notre travail, c'est déjà d'être dehors, visibles, présents, d'aller là où on nous demande, d'aller vérifier, contrôler. Des fois on n'a rien » (commandant, Préfecture de police, octobre 2012)

Néanmoins, cette rupture semble moins nette que celle apparaissant entre officiers et gradés. En effet, commissaires et officiers, en particulier ceux qui ont un rôle de responsable d'unité, ont souvent des fonctions assez analogues ${ }^{20}$. La continuité entre le corps de commandement (officiers) et celui d'encadrement et d'application (gradés et gardiens de la paix) est moins évidente. Les gradés (brigadiers, chefs ou majors) qui encadrent directement les équipes traduisent les ordres, avec tout ce que la traduction comporte de transmission et de transformation. Men in the middle, pour reprendre la formule de D. Monjardet (1996), ils jouent un rôle central dans l'ajustement entre les demandes hiérarchiques et les pratiques des agents. Ils peuvent parfois renforcer la pression sur les résultats, en posant des exigences supérieures à celles de la hiérarchie pour être certains de satisfaire cette dernière. Ils peuvent également négocier les demandes quantifiées, en soulignant qu'elles mettent en péril la sécurité des agents. Dans l'une des unités de voie publique rencontrées, gardiens de la paix comme gradés s'accordaient pour souligner l'importance donnée aux règles de sécurité aux dépens de l'atteinte d'objectifs chiffrés :

"Je dis toujours aux collègues "Je ne te jugerai jamais sur le nombre d'interpellations que tu vas me ramener dans le mois". Par contre, je les regarde sur les contrôles. Et là où j'ai une tolérance zéro, c'est s'il y a un manque de sécurité sur le contrôle » (gradé, chef de groupe de sécurité de quartier, octobre 2012).

"Il y a deux logiques qui s'affrontent et ce qui est essentiel, c'est d'avoir une hiérarchie au milieu, des brigadiers-chefs, des brigadiers qui ont le pouvoir de faire tampon et de pouvoir dire en haut "c'est bien ce que vous me dites mais sur le terrain, ça se passe peut-être différemment et les objectifs que vous donnez, sont peut-être pas réalisables ou le chiffre que vous me demandez, c'est pas viable au niveau de la sécuritê" (gardien, groupe de sécurité de quartier, octobre 2012).

Les gradés voient souvent derrière les consignes transmises par les échelons hiérarchiques supérieurs une incitation déguisée à faire du chiffre, même si ce n'est pas nécessairement le cas. Un exemple flagrant nous a été fourni dans un arrondissement parisien.

${ }^{20}$ On peut par ailleurs penser que la réforme des corps et carrières, qui a amené les officiers vers la catégorie $\mathrm{A}$ de la fonction publique a contribué à renforcer l'intégration entre officiers et commissaires. Les discussions récentes autour de la fusion de ces corps sont un signe de ce rapprochement; une telle question fait désormais partie du pensable. 
Un capitaine demandait à ses effectifs de verbaliser des jeunes qui se regroupaient sur la voie publique, afin de lutter contre les incivilités. Un brigadier se trouvant sous son autorité considérait que cette demande correspondait avant tout à des impératifs statistiques, que la verbalisation ne faisait pas partie des missions de son unité et n'a donc pas cherché à faire appliquer ces consignes par ses subordonnés. Une autre illustration nous est donnée par un Commissaire central d'arrondissement, qui a voulu mettre en place une procédure simplifiée pour traiter plus rapidement les vendeurs à la sauvette et ainsi faire gagner du temps à ses effectifs de voie publique :

"J'ai instauré ça.J'ai fait une note de service pour dire "dorénavant, sur les gars qui sont connus de vente à la sauvette, pour renforcer la présence de voie publique sur le terrain, vous avez la possibilité - ce n'est pas une obligation - de la traiter sur place, comme ça, ça vous évite de le ramener, etc.". Au début, ils ont dit "elle veut qu'on fasse du chiffre. Elle veut qu'on en fasse plus". » (août 2013).

En définitive, peu importe de savoir quelles sont les véritables finalités poursuivies dans les deux exemples cités plus haut, ce qu'ils nous montrent, ce sont les relations en définitive assez distantes et la communication difficile au sein des commissariats. Les ordres de la hiérarchie sont souvent perçus au travers du prisme froid du seul chiffre. La hiérarchie est ici discontinue, marquée par des malentendus et un faible partage des orientations. La question des résultats chiffrés constitue donc un objet de négociation, plus ou moins explicite, et une source de tension entre les différents niveaux hiérarchiques. De façon plus profonde, l'enjeu du chiffre est un point autour duquel se définissent les identités professionnelles. Au cours des entretiens, est revenue de la part des agents du corps d'encadrement et d'application, voire de certains officiers dans les unités d'investigation, l'idée que la hiérarchie travaillait d'abord pour les chiffres, là où eux font du vrai travail policier. Deux propos extraits d'entretiens avec des chefs de brigade attestent de cette représentation : "La hiérarchie, elle travaille pour ses chiffres, nous, on travaille pour les victimes »; "Le commissaire de police, ce n'est pas un flic, c'est un chef d'entreprise qui doit gérer des moyens...».

\section{Conclusion}

La diffusion des indicateurs de performance a incontestablement modifié le fonctionnement de la police nationale. Ainsi, la pression sur les résultats conduit dans certaines circonstances à redéfinir les priorités de la police, en donnant plus d'importance à ce qui peut être compté et facilement réalisé. Cependant, les critiques que génère ce type de pratiques peuvent aussi venir limiter ce type d'effet : les instruments peuvent être modifiés pour corriger les effets pervers des mesures précédentes ${ }^{21}$. Ceci a alors pour conséquence de multiplier les objectifs à atteindre (Bruno et Didier, 2013). Ce que montre notre enquête, c'est en effet une tendance à la multiplication des demandes d'indicateurs chiffrés par la hiérarchie policière, en lien avec la demande de « résultats » mais aussi pour lier ces résultats à des actions menées et des moyens disponibles.

\footnotetext{
${ }^{21}$ Ainsi, en réponse à la critique selon laquelle on peut faire monter artificiellement le taux d'élucidation en faisant des ILS et des ILE, les IRAS (infractions révélées par l'activité des services) ont été isolées des autres types d'infractions dans les comptabilisations policières.
} 
La recherche montre également que la multiplication des indicateurs chiffrés renforce la logique de centralisation organisationnelle, tant entre niveaux national et local qu'au sein des commissariats. Assignation d'objectifs, logique permanente de remontée d'information, systèmes d'incitations et de sanctions, ont contribué à renforcer l'intégration organisationnelle de la police. Malgré les annonces ponctuelles autour d'une gestion plus décentralisée de la performance, l'usage des indicateurs s'est essentiellement adossé à la logique centralisée prévalant au sein de l'institution et leur développement s'est accompagné de la mise en place de mécanismes de redevabilité interne plus forts pour les différentes composantes de l'organisation. Les tableaux de bord divers et variés remontent le long des chaînes hiérarchiques, et sont l'objet d'agrégations, d'adjonctions et d'analyses aux niveaux centraux. Ces remontées centralisées sont source de frustrations de la part d'agents qui se plaignent de devoir transmettre des données de façon répétée, sans nécessairement avoir les moyens de produire l'information demandée et sans toujours en connaître l'usage.

Pour autant, pour chacune de ces dimensions, l'épaisseur de l'organisation policière imprime une marque sur la façon dont ces outils sont mobilisés. Les réappropriations, détournements, contournements, évitements jouent à chacun des niveaux. Si centralisation il y a, celle-ci demeure imparfaite, tant elle s'oppose constamment à la complexité d'une organisation marquée par la multiplicité des zones d'incertitude internes à l'organisation. Cette étude fait apparaître une contradiction entre une volonté des échelons hiérarchiques centraux de guider et contrôler l'activité des échelons inférieurs et une capacité de ces derniers à s'arranger pour satisfaire en apparence les demandes des premiers, au prix d'un certain nombre de bricolages masquant en partie la réalité de leur action. De ce point de vue, l'usage systématisé des indicateurs révèle une forme d'hypocrisie organisationnelle (Brunsson, 2002) : s'ils peuvent apparaître comme une réponse à une exigence externe de plus grande performance (l'organisation politique dans la logique de Brunsson), leur usage démontre une relative stabilité du fonctionnement institutionnel (l'organisation d'action), même s'ils sont à l'origine de nouvelles tâches, de nouvelles pratiques d'enregistrement, et sont source de tensions supplémentaires. 


\section{Bibliographie}

Bezes P., 2012. État, experts et savoirs managériaux. Les producteurs et diffuseurs du New Public Management en France depuis les années 1970. Actes de la recherche en sciences sociales 193, 16-37.

Belorgey N., 2010. L’hôpital sous pression. Enquête sur le nouveau management public. La Découverte, Paris.

Biondi Y., Chatelain-Ponroy S. et Sponem S., 2008. De la quantification comptable et financière dans le secteur public : promesses et usages de la gestion par les résultats. Politiques et management public 26 (3), 113-125.

Bruno I., 2008. La recherche scientifique au crible du Benchmarking. Revue d'histoire moderne et contemporaine 55 (4 bis), 28-45.

Bruno I. et Didier E., 2013. Benchmarking. L’État sous pression statistique. La Découverte, Paris.

Brunsson N., 2002. The Organization of Hypocrisy $2^{\text {nd }}$ Edition. Copenhagen Business School Press, Copenhagen.

Collier P., 2006. In Search of Purpose and Priorities: Police Performance Indicators in England and Wales. Public Money \& Management 26 (3), 165-172.

Cos R., 2012. Les élus socialistes face aux chiffres de la délinquance. Dispositions, positions et prises de position partisanes sur les statistiques policières. Mots. Les langages du politique 100, 107-122.

Cour des Comptes, 2013. Police et gendarmerie nationale : dépenses de rémunération et temps de travail. Rapport public thématique.

Eterno J. et Silverman E., 2012. The Crime Numbers Game. Management by Manipulation. CRC Press, Boca Raton.

FitzGerald M., Hough M., Joseph I., Qureshi T., 2002. Policing for London. Cullompton, Willan.

Hibou B., 2012. La bureaucratisation du monde à l'ère néo-libérale. La Découverte, Paris.

Hough M., 2007. Policing, New Public Management and Legitimacy in Britain In T. Tyler (ed.), Legitimacy and criminal justice: an international perspective, The Russell Sage Foundation, London.

Jones T. and Newburn T., 2009. Le managérialisme et la nature des réformes policières en Angleterre et au Pays de Galles. Revue française de science politique 49 (6), 1175-1196.

Le Bouillonnec J.-Y., Quentin D., 2013. Mission d'information relative à la mesure statistique des délinquances et de leurs conséquences. Assemblée Nationale, Paris.

Lopez L., 2007. Faire du chiffre pour exister. Les gendarmes et les policiers au principe des statistiques judiciaires (1875-1914). Histoire et mesure 22 (2), 75-101.

Loveday B., 2006. Policing Performance: the Impact of Performance Measures and Targets on Police Forces in England and Wales. International Journal of Police Science \& Management 8 (4), 282-293.

Maillard de J., 2009. Réformes des polices dans les pays occidentaux. Une perspective comparée. Revue française de science politique 59 (6), 1197-1230.

Maillard J. de and Savage S., 2012. Comparing Performance: the Development of Police Performance Management in France and Britain. Policing \& Society 22 (4), 363-383.

Manning P., 2001. Theorizing Policing. The Drama and Myth of Crime Control in the NYDP. Theoretical Criminology 5 (3), 315-344.

Matelly J.-H. and Mouhanna C., 2007. Police. Des chiffres et des doutes. Michalon, Paris.

Marx G., 1988. Undercover: Police Surveillance in America. University of California Press, Berkeley.

Monjardet D., 1996. Ce que fait la police. Sociologie de la force publique. La Découverte, Paris.

Monjardet D., 2006. Comment apprécier une politique policière? Le ministère Sarkozy (7 mai 2002-30 mars 2004). Sociologie du travail 48 (2), 188-208.

Mucchielli L., 2008. Le «nouveau management de la sécurité » à l'épreuve : délinquance et activité policière sous le ministère Sarkozy (2002-2007). Champ pénal/Penal Field mis en ligne le 28 avril 2008. http:// champpenal.revues.org/document3663.html.

Patrick R., 2011. A Nod and a Wink: do Gaming Practices Provide an Insight into the Organizational Nature of Police Corruption. The Police Journal 84, 199-221. 
Pierru F., 2013. Les mandarins à l'assaut de l'usine à soins. Bureaucratisation néolibérale de l'hôpital français et mobilisation de l'élite hospitalo-universitaire In Hibou, B. (dir.), La bureaucratisation néolibérale, La Découverte, Paris, 203-230.

Pollitt C. and Bouckaert G., 2009. Continuity and Change in Public Policy and Management. Edward Elgar, Cheltenham.

Purenne A., Aust J., 2010. Piloter la police par les indicateurs? Effets et limites des instruments de mesure des performances. Déviance et Société 34 (1), 7-28.

Roché S., 2008. Performance Management in France: A Police or an Electoral Issue? Policing, 2 (3), 331-339.

Savage S., Golding B., 2008. Leadership and Performance Management In Newburn T. (ed) The Handbook of Policing. Cullompton, Willan. 